\title{
Kernos
}

Revue internationale et pluridisciplinaire de religion grecque antique

30 | 2017

Varia

\section{Religion and Society in Ancient Thessaly}

\section{Vinciane Pirenne-Delforge}

\section{OpenEdition \\ Journals}

\section{Édition électronique}

URL : http://journals.openedition.org/kernos/2506

DOI : 10.4000/kernos.2506

ISSN : 2034-7871

\section{Éditeur}

Centre international d'étude de la religion grecque antique

\section{Édition imprimée}

Date de publication : 1 octobre 2017

Pagination : 313-315

ISSN : 0776-3824

\section{Référence électronique}

Vinciane Pirenne-Delforge, "Religion and Society in Ancient Thessaly », Kernos [En ligne], 30 | 2017, mis en ligne le 01 octobre 2017, consulté le 23 septembre 2020. URL : http://journals.openedition.org/ kernos/2506 ; DOI : https://doi.org/10.4000/kernos.2506

Ce document a été généré automatiquement le 23 septembre 2020.

Kernos 


\title{
Religion and Society in Ancient Thessaly
}

\author{
Vinciane Pirenne-Delforge
}

\section{RÉFÉRENCE}

Maria MILI, Religion and Society in Ancient Thessaly, Oxford, Oxford University Press, 2015. 1 vol. $16 \times 24$ cm, XIV+430 p. (Oxford Classical Monographs). ISBN : 978-0-19-871801-7.

1 Autant annoncer la couleur d'emblée : il s'agit d'un excellent livre, qui vient s'ajouter à la liste déjà longue des monographies qui traitent de la vie religieuse d'une région du monde grec. Cette liste compte, entre autres, les travaux de Philippe Bruneau sur Délos, de Madeleine Jost sur l'Arcadie, de Fritz Graf sur l'Ionie septentrionale, de Robert Parker sur Athènes et l'Attique, de Katja Sporn sur la Crète, d'Irene Polinskaya sur Égine, et tout récemment le livre de Stéphanie Paul sur l'île de Cos. L'ouvrage de Maria Mili, fondé sur une thèse de doctorat soutenue à Oxford sous la direction de Robert Parker, se trouve dès lors en fort bonne compagnie, même si elle ne manque pas de critiquer certaines de ces approches régionales qu'elle trouve trop rigides. Le peu d'intérêt que ces livres portent, selon elle, à l'individu au sein de la vie religieuse de la région étudiée conduit l'A. à tenter de dépasser le catalogue sec et froid des sanctuaires et des cultes. En attendant de revenir sur ce dernier point, soulignons que l'A. offre une brique solide à l'édifice que tentent de reconstruire, en adoptant des points de vue variés, les historiens de la religion grecque.

2 Dans le cas présent, c'est la Thessalie des périodes classique et hellénistique qui est le laboratoire d'une enquête très bien informée des problématiques les plus récentes de l'étude du polythéisme grec. La plus discutée de ces questions est sans doute celle qui met en jeu les articulations entre « religion » et " société » (les deux mots-clés du titre du livre) dans le cadre du modèle de la polis-religion. Le point de vue de l'A. est clairement affirmé dès le premier chapitre qui tient lieu d'introduction méthodologique : le cadre de l'ethnos thessalien ne relègue pas la polis hors du champ de 
l'étude ; la polis religion reste une manière efficace d'interroger les questions d'autorité et de participation dans un contexte où les cités coexistent avec le cadre plus large de l' ethnos. Mais au-delà de chaque cité, il existe une tonalité religieuse proprement thessalienne, dont certains stéréotypes anciens sur la religion disent bel et bien quelque chose, en dépit de toutes leurs limites. Le reste de l'introduction permet de situer en Thessalie d'autres questionnements essentiels: l'impact du type de documentation auquel on a affaire sur les représentations religieuses que l'on peut reconstituer, l'identité des dieux au sein des panthéons locaux, les contextes sociaux spécifiques qui accueillent représentations et pratiques.

3 Le chapitre 2 (Oligarchic Constitution and Religion in the Thessalian Poleis) rencontre la nécessité d'articuler religion et société en posant le problème des structures sociales thessaliennes et de leur impact sur la vie religieuse. L'ambition clairement affichée de cette démarche est précisément de dépasser le caractère statique d'une étude centrée sur les seuls cultes et sanctuaires. Si la citoyenneté est un critère fondamental pour comprendre la vie religieuse dans l'Athènes démocratique, les constitutions oligarchiques thessaliennes imposent de penser autrement la relation entre religion et société, quand cette société est avant tout fondée sur les différences de fortune et de statut. La question de la "participation » (et notamment celle des femmes) et celle des rapports entre groupes de parenté et cité sont, selon l'A., à poser différemment en contexte thessalien. Toutefois, le caractère éclaté et fragmentaire de la documentation, dont l'A. tire tout le parti possible en posant d'excellentes questions, l'oblige tout de même à constater que "the dichotomy of oligarchy versus democracy is in some respects abstract and schematic » (p. 86).

4 Le chapitre 3 (Polis Cults) retourne la perspective en ne partant plus, cette fois, des groupes qui composent la société et de leur rapport à la polis-religion, mais en analysant l'importance des cultes de la cité pour la vie religieuse de la population. La question de la participation et de l'identité des participants à tel ou tel culte est donc centrale dans l'analyse, même si les limites de la documentation freinent les ambitions de l'A. à cet égard. La structuration du chapitre s'opère en fonction des lieux récurrents que sont l'acropole et l'agora, où les cultes attestés dans différentes cités sont analysés dans des mises au point qui englobent les cultes de ces dieux présents aussi sur le territoire des cités. Les cultes d'Asclépios et d'Ennodia, situés «around the walls» sont ensuite abordés, le culte du dieu étant réputé provenir de la thessalienne Trikka, tandis que la déesse était considérée comme la divinité thessalienne par excellence. Dans le premier cas, l'A. remarque que l'expansion du culte d'Asclépios s'est faite en Thessalie en même temps que partout ailleurs, ce qui rend décidément problématique l'identification d'une origine proprement thessalienne du dieu. Quant à Ennodia (" celle sur la route»), son portrait est ici subtilement nuancé, bien plus complexe que celui d'une divinité chthonienne des charmes magiques ou des chevaux. La conclusion du chapitre interroge l'importance symbolique des cultes dans la vie des gens, une question différente de celle de la participation aux cultes et de leur fonction. Malgré toute la compétence et la finesse d'analyse de l'A., il est vraiment difficile de dépasser le stade de quelques hypothèses qui rejoignent globalement ce que l'on pourrait affirmer de bien d'autres régions. Ce que l'A. désigne elle-même comme le palimpseste de l'image des cultes d'une cité thessalienne " théorique " ne doit certes pas conduire à oublier les identités locales, mais ne pas les oublier n'implique pas forcément de disposer des moyens pour les reconstruire. 
5 Le chapitre 4 (Thessaly through the Kaleidoscope) tente pourtant d'affronter le défi d'une attention accrue aux questions de temps et de lieux. L'option géographique adopte le canevas d'un tour imaginaire de Thessalie pour appréhender les particularités des lieux, leurs différentes "saveurs" (flavours), qui met en évidence certains cultes et sanctuaires, et les traditions mythiques qui caractérisent divers lieux ou peuvent aussi les relier. La dimension chronologique de l'enquête est quant à elle centrée sur l'étude de cas offerte par la cité de Démétrias, née à la suite d'un synécisme imposé par le pouvoir macédonien, sans négliger pour autant ce que l'on peut dire des évolutions en d'autres lieux - ou plutôt le peu que l'on peut en dire.

6 Le chapitre 5 (Panthessalianism and Religion) aborde les traditions cultuelles, ainsi que narratives qui permettent d'appréhender une «identité thessalienne » telle qu'elle était vue par les Thessaliens eux-mêmes. Le parcours à travers différents cultes fédérateurs et les mythes étiologiques qui s'y accrochent, l'A. identifie un " panthessalianisme » qui excède les moments fugaces où le koinon thessalien présente une relative unité. L'identité en question dépasse les manipulations momentanées à des fins politiques. Il s'agit d'un courant puissant qui oscille entre différents pôles comme l'autochtonie ou l'histoire de la conquête militaire de la région, mais sans jamais se limiter à l'un ou à l'autre.

7 Le chapitre 6 (The Land Rich in Herbs) parle également d'image et d'identité, mais celle qui est construite par les autres, cette fois, et tout particulièrement par les traditions athéniennes. Chevaux, prairies, plantes et un sens de la fête opulente en composent l'arrière-plan sur lequel la vie religieuse est vue au prisme d'une prétendue obsession de la mort et de la magie. Ces stéréotypes, véhiculés dès l'antiquité, ont été largement assumés dans les travaux modernes et ce sont eux que l'A. s'attache à poser et puis à déconstruire au profit des traits centraux de l'identité thessalienne que sont la philoxenia et les rites d'hospitalité.

8 Après un bref Epilogue qui affronte une dernière fois les questions posées en introduction, l'ouvrage regroupe de précieux appendices : un tableau (474 entrées) des dédicaces inscrites ordonnées par ordre alphabétique des dieux auxquels elles s'adressent, une présentation résumée des sanctuaires thessaliens fouillés, un catalogue toponymique des groupes «socio-religieux» attestés dans la documentation épigraphique. Une bibliographie et un index général referment cet ensemble très réussi, fondé sur une documentation remarquablement exploitée, notamment le foisonnement des rapports de fouille. L'A. fait preuve d'une remarquable maitrise, à la fois des corpus en jeu et des multiples questions à leur poser. Elle manifeste une capacité tout aussi remarquable à ordonner dans un tableau d'ensemble une documentation parcellaire, fragmentée, difficile. Il faut saluer les efforts consentis pour dépasser le seul catalogue des cultes et sanctuaires, qui est le refuge de nombre d'études régionales confrontées à l'éclatement de la documentation. Le tableau de la vie religieuse thessalienne qui ressort de ce livre est assurément plus vivant que nombre d'études antérieures. Mais la qualité des questions posées et la maîtrise du matériel existant ne suffisent pas pour appréhender les individus eux-mêmes dans leurs représentations et leurs pratiques religieuses. Cette intention liminaire n'aboutit pas et il faut probablement s'interroger sur la pertinence de la question elle-même, que soulèvent continument des travaux récents sur les polythéismes antiques. Quoi qu'il en soit de ce point, l'ouvrage est un modèle d'étude régionale de la religion grecque antique et un passage désormais obligé sur la Thessalie en général. 


\section{AUTEURS}

VINCIANE PIRENNE-DELFORGE

F.R.S.-FNRS - Université de Liège 